\title{
Profil des patients ayant une dysfonction sexuelle masculine à l'hopital général de Yaounde
}

\author{
F. F. ANGWAFO III*., E. EIMO MALONGA, M. POYI KAMDEM \\ Faculté de Médecine et des Sciences Biomédicales ; Université de Yaoundé I - Cameroun
}

\begin{abstract}
Le mythe selon lequel les Africains mâles ont des prouesses sexuelles exceptionnelles est bien connu. Il est donc impensable qu'ils puissent souffrir de dysfonction sexuelle masculine (DSM). Une enquête pilote sur la DSM et les causes du symptôme dysfonction érectile (DE) dans un groupe de patients hospitaliers a été menée.

Entre février 1997 et mars 1999, 166 hommes ayant une DSM ont été recrutés en série en consultation uro-andrologique à l'Hôpital Général de Yaoundé. Chaque patient a bénéficié d'une histoire complète, d'un examen physique et d'un bilan minimum.
\end{abstract}

RESUME

L'age moyen des 166 hommes répertoriés était de 42,93 ans, avec des extrêmes de 19 à 81 ans. 88 patients $(53 \%)$ avaient 40 ans ou plus. 150 $(90,36 \%)$ souffraient d'une DE depuis une durée d'au moins 6 mois. $38(22,89 \%)$ présentaient une anxiété de performance; 30 $(18,07 \%)$ avaient des troubles de l'éjaculation (26 éjaculations précoces et 4 éjaculations rétrogrades); $22(13,25 \%)$ avaient une anxiété de désir; 18 avaient une perte de libido.

Les prestations sexuelles antérieures de ces patients ont été décrites en terme quantitatif: $64,3 \%$ avaient moins de 3 érections par semaine. La description qualitative de la fonction érectile a révélé que les causes de DE étaient mixtes $(40,5 \%)$, organiques $(36 \%)$ et psychogènes $(23,5 \%)$.

Les maladies associées aux DE organiques étaient le diabète $(15,6 \%)$, l'hypertension $(8,4 \%)$ et l'athérosclérose $(6,0 \%)$. La DE psychogène était surtout en rapport avec la peur des maladies sexuellement transmissibles, un conflit familial ou conjugal, très souvent lié à l'infertilité du couple, l'infidélité, les difficultés financières et professionnelles et le stress.

La présente étude écarte le mythe selon lequel les aptitudes érectiles et les performances sexuelles de l'Africain sont liées à sa race. Les Africains souffrent de DE. Les pharmacopées traditionnelles ne traitent pas adéquatement la DE.

Mots clés: Dysfonction érectile, africain noir, causes, mythe

\section{INTRODUCTION}

Le mythe selon lequel les Africains mâles ont des prouesses sexuelles exceptionnelles est bien connu. Il est donc impensable qu'ils puissent se présenter à l'hôpital avec une dysfonction sexuelle battant en brèche les idées reçues.

Correspondance: Faculté de Médecine et des Sciences Biomédicales Université de Yaoundé I CAMEROUN. Tel: 237-20-28-21. e-mail: fobuzshi@yahoo.com ou asanji25@hotmail.com. 
Pourtant les Africains mâles doivent souffrir de dysfonction sexuelle; consultent les tradipraticiens qui proclament des guérisons miraculeuses à partir des principes phytothérapeutiques, des cornes de rhinocéros, et des médications diverses et variées.

Le Massachusetts Male Aging Study (MMAS) publié par Feldman en 1994 et la découverte du Sildénafil, une pilule facile à prendre et efficace sur la dysfonction érectile, a rallumé l'intérêt médical dans ce domaine [4]. Bien que jusqu'ici aucune médication orale n'ait été aussi efficace que le Sildénafil [8]. Le coût est une limitation significative dans les communautés subsahariennes pauvres. Ceci a attisé une anxiété considérable dans la communauté, suscitant des attitudes variées sur son utilisation.

Les critiques de l'étude de Feldman faisaient remarquer que, compte tenu de la structure différente de la population, des différences culturelles et du mythe des prouesses sexuelles de l'Africain, les résultats de Massachussets ne pouvaient être extrapolés aux communautés sub-sahariennes.

Il était donc utile qu'une étude de base "culturellement appropriée" soit menée sur le profil des hommes ayant une dysfonction érectile (DE).

Le traitement de la DE serait alors plus efficace si ses causes étaient mieux déterminées.

Il s'agit d'une enquête pilote sur les causes du symptôme D.E dans un groupe de patients hospitaliers.

\section{PATIENTS ET METHODES}

L'Hôpital Général de Yaoundé est un centre de référence de soins tertiaires servant la population de la capitale du Cameroun. Il est situé dans la province centrale du Cameroun avec une population cible de 2 millions d'habitants.

Néanmoins, il reçoit les malades de tout le pays et de la sous région Afrique Centrale.

C'est une institution où les services sont payés par une population sans assurance-maladie. L'accès à l'hôpital a des limitations financières. En plus, bien qu'il s'agisse d'une ville cosmopo- lite, l'accès à l'hôpital est limité par les barrières culturelles.

Le recrutement en série de tous les patients ayant une dysfonction sexuelle mâle a été mené à l'Hôpital Général de Yaoundé en consultation uro-andrologique pendant une période de deux (02) ans.

Chaque patient avait une histoire complète incluant ses antécédents sexuels, maritaux, familiaux, sociaux et professionnels. Afin de tester le degré de compréhension de l'IIEF, chez nos patients, un exemplaire de l'IIEF a été remis à chacun d'eux. L'examen physique complet était fait et l'impression diagnostique posée.

Tous les patients bénéficiaient d'une Numération Formule Sanguine (NFS), d'une Vitesse de Sédimentation (VS), et d'une glycémie. Les autres bilans étaient demandés en fonction de l'orientation clinique et étiologique notamment l'électrophorèse de l'hémoglobine la testostéronémie, la prolactinémie, des tests de la fonction hépatique et rénale et une évaluation Doppler de l'aorte et du pénis. Quelques patients avec nodules péniens avaient des études Echo-Doppler du phallus.

Les données collectées étaient introduites dans un répertoire de patient individuel créé dans le logiciel épi info $5.1 \mathrm{du}$ CDC, Atlanta, Georgia. Une étude descriptive des variables épidémiologiques (fréquences, histogrammes, etc.) était faite.

\section{RESULTATS}

Entre février 1997 et mars 1999, 166 hommes se sont présentés à l'Hôpital Général de Yaoundé avec une Dysfonction Sexuelle Mâle (D.S.M.). L'âge moyen était de 42,93 ans, DS (Déviation Standard) de 14,02 ans, avec des extrêmes de 19 à 81 ans.

- 88 (53\%) patients avaient 40 ans ou plus (Figure 1). Aucun patient n'était musulman. Tous étaient chrétiens.

- 150 sur 166 hommes $(90,36 \%)$ souffraient d'une DE depuis une durée d'au moins 6 mois;

- $38(25,3 \%)$ présentaient une anxiété de performance; 
- 30 sur 166 hommes $(18,07 \%)$ avaient des troubles de l'éjaculation (26 éjaculations précoces et 4 éjaculations rétrogrades);

- 22 sur 166 hommes (13,25\%) avaient une anxiété de désir;

- 18 sur 166 hommes $(10,84 \%)$ avaient une perte de la libido.

Les prestations sexuelles antérieures de ces patients ont été décrites en terme quantitatif:

- $64,3 \%$ avaient au moins 3 rapports sexuels par semaine quand ils étaient en bonne santé sexuelle;

- $21,1 \%$ avaient une érection et des rapports sexuels désirés à tout moment;

- $14,59 \%$ avaient moins de 3 érections par semaine.

La description qualitative de la fonction érectile permettait d'établir une distinction clinique entre $\mathrm{DE}$ psychogène et $\mathrm{DE}$ organique.

Les érections non soutenues ou faibles étaient plus fréquentes chez les patients souffrant d'une DE organique.

Les érections étaient de bonne qualité, lorsqu'elles survenaient chez les patients souffrant d'une $\mathrm{DE}$ psychogène.

- 40,5\% des causes de DE étaient mixtes (organiques et psychologiques);

- $36 \%$ des causes étaient organiques;

- $23,5 \%$ des causes étaient psychogènes.

Les maladies associées aux DE organiques étaient le diabète $(15,6 \%)$, l'hypertension $(8,4 \%)$ et l'athérosclérose $(6,0 \%)$ (Tableau 1$)$. Lâge moyen des patients qui avaient une DE organique était de 43,5 ans avec des extrêmes de 21 et 71 ans.

La DE psychogène était surtout en rapport avec la peur des maladies sexuellement transmissibles, un conflit familial ou conjugal, très souvent lié à l'infertilité du couple, l'infidélité, les difficultés financières et professionnelles et le stress dans l'environnement social.

Lâge moyen des patients qui souffraient d'une DE psychogène était de 37,6 ans avec des extrêmes de 19 à 81 ans.
Plusieurs patients avaient un mélange de symptomatologie simultanément : 13 sur 166 $(7,8 \%)$ avaient une DE et une perte de la libido. Leurs âges variaient de 19 à 71 ans.

$18(10,9 \%)$ avaient une DE et des troubles de l'éjaculation associés. Aucun patient n'avait une anéjaculation dans cette série. $31(18,7 \%)$ patients avaient une anxiété de performance et $17(10,2 \%)$ une anxiété de désir associée à une DE.

\section{DISCUSSION}

Avant l'étude menée et publiée au Massachussets sur la DE en 1994, la dysfonction sexuelle demeurait un tabou dans plusieurs parties du monde y compris l'Afrique au Sud du Sahara. Très peu sont les institutions médicales qui ont un cours formel sur la fonction sexuelle dans leur curriculum médical.

Cependant, c'est un vieux problème humain dont les répercussions inhérentes sont lointaines : conflits conjugaux, états de dépression et baisse de la productivité au lieu du travail.

On estime que 10 à $30 \%$ des hommes souffrent de dysfonction érectile (DE) [6]. Ce pourcentage augmente de manière significative pour atteindre entre 30 et $52 \%$ chez les hommes de plus de 40 ans $[4,8]$. Ces statistiques viennent des sociétés les plus ouvertes, où $55 \%$ de patients souffrant de DE ne sont pas au courant des solutions thérapeutiques. En plus, le manque de connaissances de la part des communautés médicales et le caractère tabou du sujet, exacerbaient l'anxiété du patient [5]. Ainsi, moins de $1 \%$ des patients souffrant de DE recherchaient une aide médicale [4].

Le patient africain souffrant de DE se trouvait en soi dans un dilemme puisqu'il était admis que ses aptitudes érectiles et ses performances sexuelles étaient liées à sa race. La présente étude écarte ce mythe et montre que non seulement les Africains souffrent de DE, mais aussi que les pharmacopées traditionnelles ne traitent pas adéquatement la $\mathrm{DE}$.

Cette étude a été menée au moment où l'évaluation paraclinique du dysfonctionnement n'était pas encore très élaborée.

Le diagnostic de $\mathrm{DE}$ n'était pas posé à partir de l'IIEF (Indice Internationale de la Fonction Erectile) pour plusieurs raisons. 
Tableau 1 : Les causes de dysfonction sexuelle mâle.

Causes

Nombre de patients

Les causes psychogènes pures

Les causes organiques

Maladies cardio-vasculaires

Hypertension artérielle

Artériosclérose

Maladies vasculaires périphériques

Diabète

Autres*

Hypogonadisme

Chirurgie pelvienne

Médicaments

Insuffisance hépatique

Maladies sexuellement transmissibles

Maladies de la Peyronie

Fractures pelviennes

Maladies neurologiques

Hyperprolactinémie

39

127

31

14

10

7

26

15

9

7

7

7

7

6

4

4

4
Pourcentage

$23,5 \%$

$76,5 \%$

$18,67 \%$

$8,43 \%$

$6,02 \%$

$4,22 \%$

$15,66 \%$

$9,03 \%$

$5,42 \%$

$4,2 \%$

$4,2 \%$

$4,2 \%$

$4,2 \%$

$3,6 \%$

$2,4 \%$

$2,4 \%$

$2,4 \%$

TO'TAL

* Cigarettes, l'insuffisance rénale, l'insuffisance cardiaque, l'alcoolisme, maladies de la prostate et la dépression.

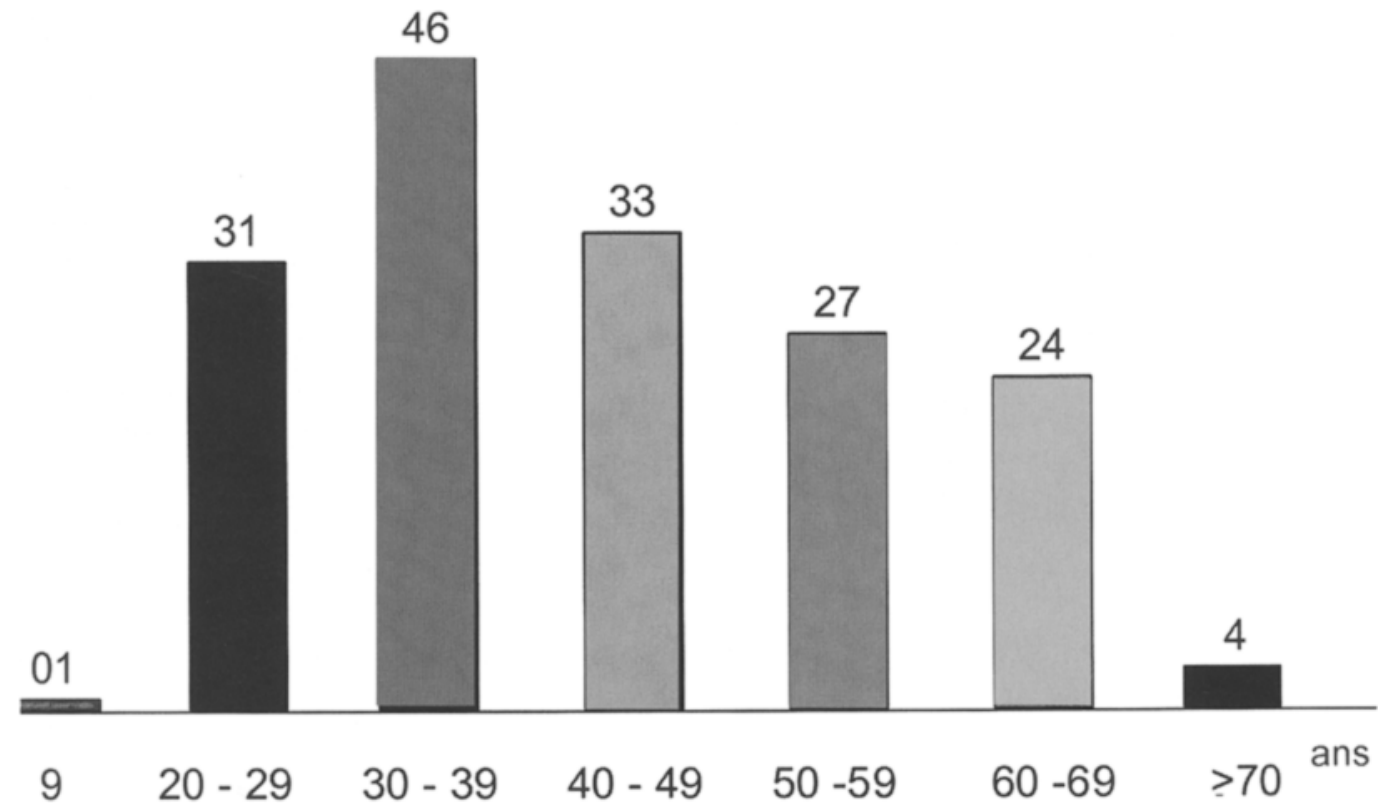

Figure 1 : Histogramme âge 
Premièrement, dans cette forme de questionnaire auto-administré le patient doit être lettré et avoir un niveau de compréhension. $76 \%$ de ces patients ne comprenaient pas assez bien les questions de l'IIEF pour répondre avec pertinence.

En plus l'IIEF n'a pas été traduit dans les communautés. Il n'a pas été testé et validé si l'IIEF est "culturellement approprié" dans la communauté concernée. La population étudiée n'est pas aussi représentative de la population générale. L'IIEF a été testé et validé ailleurs [1]

Autrefois, on enseignait que la majorité de DE étaient de causes psychogènes [7]. Ceci n'est plus le cas dans la plupart de cas.

La connaissance de la physiopathologie de plusieurs maladies et la physiologie de la fonction sexuelle ont contribué à la bonne compréhension du symptôme.

$\mathrm{Au}$ fur et à mesure que la population vieillit de par le monde, l'incidence des maladies cardiovasculaires et du diabète augmente aussi et sont responsables de l'incidence croissante des dysfonctions érectiles. Même dans cette population relativement jeune, les causes organiques n'étaient pas rares mais surpassées par les causes mixtes, de loin les plus fréquentes.

\section{REFERENCES}

1. ACKERMAN M.D, D'ATTILIO J.P., ANTONI M.H., RHAMY R.K., WESTEIN D. et POLITANI V.A.: Patient reported erectile dysfonction : a cross-validation study. Arch. Sex. Beth., 1993, 22 : 603-618.

2. BRUNETT A.L.: Erectile dysfunction : a practical approach for primary care. Geriatrics., 1998, 58:3448.

3. BURNETT A.L.: New options for erectile dysfunction. Clin. Rev., 1998, (Suppl. 6): 3-7.

4. FELDMAN H.A.: Impotence and its medical and psychosocial correlates: Results of Massachusetts Male Aging Study. J. Urol., 1994, 151:54-61.

5. GOLDSTEIN I.: Oral sildenafil in the treatment of erectile dysfunction. NEJM, 1998, 338: 1397-1404.

6. GUILIAN F.: Pharmacologie périphérique de l'érection. Progrès Urol., 1997, 7:24-33.

7. National Institutes of Health Consensus Development Conference Statement: Impotence. NIH Consensus Statement. On line. 1992, 10(4): 1-31.

8. TERRETT N.K.: Sildenafil (Viagra), a potent and selective inhibitor of type 5 GMP. Phosphodiesterase with utility for the treatment of male erectile dysfunction Bioorg. Med. Chem. Letters. 1996, 6(15): 1819-1824.

\begin{abstract}
The Profile of Patients with Male Sexual Dysfunction at The Yaoundé General Hospital.
\end{abstract}
F. F ANGWAFO III., E. EIMO MALONGA., M. POYI KAMDEM.

The myth that African men have exceptional sexual prowess is well known. It is therefore unthinkable that they suffer from male sexual dysfunction (MSD).

A pilot study of MSD was carried out between February, 1997 and March, 1999. 166 men presenting with male sexual dysfunction were recruited in the Uro-andrology consultation clinic at the Yaoundé General Hospital. Each patient had a complete history; physical examination and a minimum work up.

The average age was 42,93 years, with a range from 19 to 81 years. $88(53 \%)$ patients were 40 years or over. $150(90,36 \%)$ complained of erectile dysfunction of at least six months duration. $38(22,89 \%)$ had performance anxiety; 30 $(18,07 \%)$ ejaculatory dysfunction; $22(13,25 \%)$ desire anxiety and $18(10,84 \%)$ had no libido. The sexual habits of these patients before they fell ill were quantified thus: $64,3 \%$ had sexual congress at least 3 times weekly, 21,1\% had erections and sexual intercourse on demand and $14,59 \%$ had sexual congress less than three times weekly. The qualitative description of erectile function revealed that $40,5 \%, 36 \%$ and $23,5 \%$ of the patients had ED of mixed, organic and psychogenic causes respectively.

Diabetes $(15,6 \%)$, hypertension $(8,4 \%)$ and atherosclerosis were the commonest illness associated with ED. Psychogenic ED was often associated with fear of sexually transmitted disease, marital or family conflict, often related to couple infertility, infidelity, financial and professional difficulties, and stress.

The present study dismisses the myth that the African has extraordinary erectile and sexual prowess. Africans too suffer from ED and MSD. Traditional African pharmacopoeia does not adequately treat ED and MSD.

Key words: Erectile dysfunction, African black, myth, causes 